\title{
Impact of an HIV Prevention Intervention on Condom Use among Long Distance Truckers in India
}

\author{
Sachin Juneja • Vasudha Rao Tirumalasetti • \\ Ram Manohar Mishra · Shekhar Sethu • \\ Indra Ramyash Singh
}

Published online: 25 September 2012

(C) The Author(s) 2012. This article is published with open access at Springerlink.com

\begin{abstract}
This paper examines the impact of three components of an HIV prevention program (mid-media, interpersonal communication, and project-run clinics) on consistent condom use by long distance truckers with paid and non-paid female partners in India. Data from 2,723 long distance truckers were analyzed using the propensity score matching approach. Based on utilization of services, the following categories of intervention exposure were derived: no exposure, exposure only to mid-media, exposure only to midmedia and interpersonal communication, exposure only to mid-media and project-run clinics, and exposure to all three intervention components. Compared to those who were not exposed to any intervention, exposure to mid-media alone increased consistent condom use with paid female partners by about ten percent. Exposure to mid-media and visits to project-run clinics increased consistent condom use with nonpaid female partners by $26 \%$. These findings suggest that mid-media events and clinics were the most effective package of services to increase consistent condom use among the long distance truckers.
\end{abstract}

Keywords Truckers - Intervention - Propensity scores · HIV

S. Juneja · V. Rao Tirumalasetti · I. R. Singh

Transport Corporation of India Foundation, Gurgaon, India

R. M. Mishra ( $₫)$

HIV \& AIDS Program, Population Council, 142, First Floor, Golf Link, New Delhi 110003, India

e-mail: rammanohar7@yahoo.co.in

S. Sethu

Family Health International, New Delhi, India

\section{Introduction}

Long distance truck drivers and their helpers (hereafter referred to as truckers) are considered to be at high risk for sexually transmitted infections (STI) and HIV in many parts of the world, including Asia [1-4], Africa [5-8], South America $[9,10]$ and the United States $[11,12]$. Truckers' increased HIV risk is largely attributed to long periods of absence from the family [11, 13, 14].

In India, recent evidence shows that around one-fourth of truckers have sex with female sex workers (FSWs) [15] and less than three-fourth use condoms consistently in sex with these partners [14-16]. Substantial proportions of truckers also have sexual relations with non-paid female partners [14-16] and only about one-third use condoms consistently in such sexual encounters $[15,16]$. Studies in the country have found high HIV prevalence $(2-16 \%)[14,15,17,18]$ and high STI prevalence (3-13\%) among truckers $[15,17,18]$.

Due to high risk behaviours, high STI and HIV prevalence, and ability to transmit infections to new geographic areas, HIV prevention interventions among truckers started in early 1990s with aim to raise the awareness through group discussion on matters related to HIV, using posters and pamphlets with positive AIDS messages, street theatres and puppetry, and distribution of condoms [19-21]. Some interventions also used one-to-one and one-to-group education sessions through peers, and provided treatment for STI at truckers' halt points [22, 23]. The HIV prevention efforts among truckers were strengthened towards the end of 1990 s when a large-scale HIV prevention program among truckers, named as 'Healthy Highway' was initiated. The project offered services including treatment and counseling for STIs, condom promotion, dissemination of educational materials, and face-to-face behavioural change communication to truckers at major halt points where at 
least 50 trucks were parked at any time of the day or night [24]. In 1998, the second phase of the National AIDS Control Program initiated specific interventions among truckers that were largely based on the strategies of the Healthy Highway program [25]. The Healthy Highway program was handed over to the government in 2000 which replaced the central management team of the Healthy Highway program by independent State AIDS Control Societies [26].However, despite several individual HIV prevention programs for truckers being implemented through State AIDS Control Societies, the need for a largescale intensive program was recognized due to lack of coordination among interventions within or across states, language barriers, and the absence of a standard network of services to address the needs of truckers [26]. In this context, a large-scale intervention, called Kavach (meaning 'shield' in Hindi/Sanskrit) for truckers in India was started as part of Avahan, the India AIDS initiative [27]. In addition to working with truckers, Avahan supported interventions among other high risk groups such as FSWs and their clients, men who have sex with men/transgenders and injecting drug users in several high HIV prevalence states of India [27, 28]. This paper describes the Kavach intervention and examines the impact of its components on condom use behavior by truckers with non-regular female sexual partners.

\section{Kavach Intervention}

The Kavach intervention was initiated in 2004 in 36 transshipment locations across India covering the major national highways along six routes, namely, North-East, North-West, North-South, West-South, South-East and West-East. Transshipment locations are places where transporters and brokers operate to link truckers with clients who need to transport goods, and routes are road corridors on which truckers travel.

The Kavach intervention was redesigned in 2006 by halving the number of implementation sites from 36 to 17 to focus on the major truck halt points in nine Indian states. Unlike the other HIV prevention programs to cover large number of intervention sites for truckers coverage, the redesigned program aimed to provide maximum coverage to truckers by working with high intensity program (i.e., ensuring more contacts by peer educators, greater program visibility and increased number of project-run clinics) at major transshipment locations in the country where large number of truckers halt and wait for their consignments.

The Kavach intervention had three main components: mid-media events; peer-led dialogue based interpersonal communication (IPC) and project-run clinics. Mid-media events included street plays, health games, film shows, truckers' festivals and the distribution of audio cassettes/ compact discs. Mid-media events aimed to communicate messages on safe sex practices to truckers and motivate them to visit project-run clinics. Trained professionals conducted about ten street plays every month at each intervention site. Health games were conducted twice a day and film shows were organized once a week at each intervention site. Truckers' festivals were large-scale events conducted annually to create an interest among brokers, transporters and truckers in the program. Audio cassettes/compact discs with catchy local songs interspersed with spoofs on popular film actors delivering HIV prevention messages and endorsing services at project-run clinics were distributed to truckers so as to reinforce positive messages on HIV when they travel on the highway. The project monitoring data indicate that nationally around 150,000 audio cassettes/compact discs were distributed every year.

In the Kavach intervention, IPC included one-to-group discussions by trained peer educators who were either active truckers or ex-truckers. About 10-12 IPC sessions were conducted every day at each intervention site. In order to reinforce messages already received in the midmedia events, those messages were repeated during the IPC sessions.

One static clinic and two mobile clinics were established at each intervention site to provide services at the door-step of truckers' halting points. To avoid becoming stigmatized as STI clinics, the project clinics treated a range of general health ailments in addition to providing counseling services by a qualified counselor on safe sex practices, correct and consistent condom use, and diagnosis and treatment of STI so that truckers would not hesitate to visit these clinics. Following the McDonald's business franchisee model, where the look and service of outlets do not differ across any location [29], project-run clinics were branded with uniform appearance and services across all intervention sites to facilitate greater acceptance and easy recognition by truckers. Social marketing of condoms was promoted in partnership with condom marketing organizations by opening non-traditional outlets such as at tea stalls, and installing condom vending machines at strategically chosen places in the implementation sites. Details of the Kavach intervention are available elsewhere [26].

\section{Methods}

Study Setting and Sampling

Of the 17 intervention sites, six were purposively chosen as survey sites for data collection. The selected sites were 
located at Ghaziabad and Delhi in northern India, Indore in central India, Mumbai and Pune in western India, and Bengaluru in southern India. These sites were chosen because truckers from different parts of the country wait at these places for a considerable length of time for new consignments. Data were collected during February-August 2009 in two phases at each selected site in order to cover wider group of truckers' population. A total sample size of 2,800 truckers was estimated for the survey.

The total sample size was distributed across the six transshipment locations chosen for the survey in proportion to the number of truckers available at those transshipment locations. Parking areas for trucks were considered as clusters in each transshipment location for the multistage cluster sampling and all clusters were selected for the survey. The registration number of the trucks parked in each cluster on the day of the survey was listed and a systematic random sampling approach was used to select the trucks. The main driver or helper of the selected trucks was approached to participate in the survey.

Respondents were selected only if they met the following three criteria: (i) aged 18 years or more; (2) had worked as a trucker for the past 2 years or more; and (3) were working in a truck with a national permit (i.e., permission from the government to carry goods to and from any part of the country). Truckers who had already participated in the survey (either in an earlier phase of the survey or in the same phase) were not interviewed again.

A pre-coded questionnaire translated into the local language was used to collect data by trained native speakers. A total of 2,810 truckers were interviewed in the survey. Of these, cases who were traveling on the South-East route [30]; cases exposed only to IPC [20] and exposed only to project-run clinics [31]; and cases where there was incomplete information on exposure to intervention [2] were excluded from the analyses due to the small number of observations. This resulted in a total analytical sample of 2,723 cases.

\section{Ethical Considerations}

A comprehensive informed consent process was followed. Respondents were informed about the study including the duration of the interview (approx. $30 \mathrm{~min}$ ), and their queries addressed before written consent was taken. To protect confidentiality all questionnaires were entirely anonymous and the names and addresses of respondents were not recorded. To maintain privacy, interviews were either conducted inside the truck or in a secluded public area such a corner road where others would not be able to listen to the interview. Participants were not given any monetary compensation for their time in the study.
Measures

\section{Socio-Demographic Characteristics}

The socio-demographic characteristics considered in this paper were age (completed years), formal schooling (no, yes), marital status (not currently married, currently married), usual place of residence (native place, others), route generally travelled (North-East, North-South, North-West, West-South and West-East), duration of work as a trucker (completed years), occupation (driver, helper) and availability of music player in the truck (no, yes). Formal schooling was defined as the ability to both read and write.

\section{Exposure to the Intervention}

Exposure to mid-media was defined as exposure to at least one mid-media events (watched street plays, participated in health games, watched film shows, participated in truckers' festivals, received audio cassettes/compact discs) in the past 12 months (no, yes). Exposure to IPC was defined as participation in at least one IPC session in the past 12 months (no, yes). Exposure to project-run clinics was defined as at least one visit to a project clinic (either static or mobile) in the past 12 months (no, yes). Exposure to any intervention was defined as exposure to at least one of the three intervention components, namely, mid-media, IPC and project-run clinics in past 12 months.

To measure exposure to different intervention components in the past 12 months, a variable with the following five mutually exclusive categories were derived: no exposure, exposure only to mid-media, exposure only to midmedia and IPC, exposure only to mid-media and project-run clinics, and exposure to all three intervention components.

\section{Consistent Condom Use}

Consistent condom use with paid female partners and nonpaid female partners was the primary outcome variable measuring HIV-related risk behaviour. A paid female partner was defined as a woman to whom the respondent had paid cash in exchange for sex. A non-paid female partner was defined as a woman to whom the respondent was not married and did not pay cash in exchange for sex. Consistent condom use with a sexual partner was defined as condom use in every sexual encounter with that particular partner (no, yes).

\section{Statistical Analyses}

Basic descriptive statistics (i.e., proportions, means and standard deviations) were presented to describe participants' socio-demographic characteristics, exposure to the 
intervention, and outcome indicators. The bivariate association between the categories of intervention exposure and outcome indicators was examined using $\chi^{2}$ test.

The propensity score matching (PSM) approach was adopted to examine the impact of truckers' exposure to any intervention as well as exposure to different categories of intervention on consistent condom use by them with paid and non-paid female partners. Studies indicate that when only observational data are available without a valid control group, the PSM approach can be adopted to evaluate the impact of intervention exposure on an outcome [30-34]. The PSM approach to examine the impact of intervention exposure on consistent condom use included two steps. First, for each respondent a propensity score was calculated using multivariate logistic regression, with variable measuring exposure to intervention as the binary dependent measure and observed socio-demographic characteristics as covariates. The next step consisted of matching exposed and unexposed truckers with similar propensity scores.

The observed socio-demographic characteristics including age, literacy, marital status, occupation, duration of work as trucker, time away from family, and route category were considered as the covariates because these characteristics have been found to correlate with truckers' sexual risk behaviors in India [11, 14, 17, 35, 36]. We added presence of music player in the truck as one of the covariates as it was likely to affect truckers' exposure to mid-media intervention components (distribution of audio-cassettes/compact discs).

Matching was carried out using the radius method in which each exposed trucker was matched only with the unexposed truckers whose propensity score fell into a predefined neighborhood of the estimated propensity score of the exposed trucker [37]. The predefined neighborhood was determined by estimating the radius as one-fourth of the standard deviation of the corresponding propensity scores [37-39]. The common support restriction (to exclude data from exposed truckers with a propensity score higher than that of any unexposed trucker) was imposed to improve the quality of the matches [30]. Among unexposed truckers, those who had propensity scores similar to exposed truckers were termed as matched unexposed and they served as controls for the exposed group. Matching was done with replacement so that one matched unexposed (or exposed) trucker could potentially serve as match for several exposed (or unexposed) truckers [37].

The key assumption in the PSM approach is that conditional on the propensity score, assignments to exposed and unexposed groups can be considered as random [32]. One test of this assumption is to examine the balancing property which states that, conditional on the propensity scores, the distribution of confounding factors are similar among exposed and matched unexposed groups [38]. To examine whether the balancing property was satisfied, differences in the socio-demographic characteristics of exposed truckers with all unexposed truckers (to assess the differences before matching) and matched unexposed truckers (to assess the differences after matching) were tested for significance using the $\chi^{2}$ test (for percentages) and unpaired $t$ test (for mean values). The overall covariate imbalance of the model was examined by testing the joint significance of all the regressors (ability of covariates to predict exposure to any intervention) using the likelihood ratio test before and after matching.

The PSM approach enabled us to estimate the average effect of intervention exposure among exposed truckers (difference in condom use among exposed truckers and matched unexposed truckers) and the average effect of intervention exposure among unexposed truckers (difference in condom use of unexposed truckers and matched exposed truckers). The average effect of intervention exposure among exposed truckers measured the impact of intervention on exposed truckers whereas the average effect of intervention exposure among unexposed truckers measured the impact that the interventions would have had on unexposed truckers if they were exposed. These two effects were weighted by the proportion of truckers exposed and unexposed respectively to arrive at the impact of intervention exposure on consistent condom use which measured the increase in consistent condom use due to intervention exposure [30, 32]. As a final diagnostic check, sensitivity analyses were conducted to assess the potential effect of unmeasured confounders on the results. The Rosenbaum bounds approach was adopted, which is described elsewhere [32].

Following these steps, we first examined the impact of exposure to any intervention on condom use by comparing the consistent condom use among truckers exposed to any intervention with that among matched unexposed truckers. Since exposure to mid-media was common among all the exposed truckers, categories with significant impact on outcomes were examined against exposure only to midmedia for assessing the additional impact different components. Analyses were conducted using the statistical software STATA (version 11).

\section{Results}

Socio-Demographic Characteristics, Exposure to Intervention, and Sexual Behavior

Mean age of the respondents was about 30 years. Of the 2,723 respondents, more than three-fourth $(83.4 \%)$ had formal schooling, nearly two-third $(66.6 \%)$ were currently married, and about one-fifth $(22.1 \%)$ were working as helpers. A total of 991 (36.4\%) truckers were not exposed to 
the intervention in the past 12 months, 651 (23.9\%) were exposed only to mid-media, $188(6.9 \%)$ were exposed only to mid-media and IPC, $449(16.5 \%)$ were exposed only to mid-media and clinics, and $444(16.3 \%)$ were exposed to all three intervention components. About $45 \%$ of respondents reported sex with paid female partners in the past 12 months and almost three-fourths (74.1\%) reported using condoms consistently in such sexual encounters. About $22 \%$ of reported having sex with non-paid female partners and less than half $(41.8 \%)$ had used condom consistently in such sexual acts (Table 1).

\section{Intervention Exposure and Consistent Condom Use}

Consistent condom use with both paid and non-paid female partners was significantly higher among truckers exposed to any intervention than those with no intervention exposure (paid female partners: $76.5 \%$ vs $65.8 \%, \chi^{2}$ statistic $=12.85, p<0.001$; non-paid female partners: $45.3 \%$ vs $32.5 \%, \chi^{2}$ statistic $=7.82, p=0.005$ ) (Table 2). Consistent condom use with paid female partners varied significantly from about two-thirds $(63.3 \%)$ among truckers exposed only to mid-media to more than three-fourth $(80.7 \%)$ among those exposed only to mid-media and project-run clinics $\left(\chi^{2}\right.$ statistic $\left.=23.56, p<0.001\right)$. Consistent condom use with non-paid female partners also varied from about one-third (31.8\%) among truckers exposed only to mid-media and IPC, to almost half among those exposed to mid-media and project-run clinics $(54.9 \%)$ and those exposed to all three intervention components (50.9\%) $\left(\chi^{2}\right.$ statistic $\left.=24.67, p<0.001\right)$.

\section{Matching of Exposed and Unexposed Truckers}

Among truckers who had sex with paid female partners, those exposed to any intervention differed significantly from all unexposed truckers in terms of almost all the sociodemographic characteristics (Table 3). However, no significant differences in socio-demographic characteristics were found when truckers exposed to any intervention were compared with matched unexposed truckers. Similar results were observed for truckers who reported sex with non-paid female partners. The likelihood ratio Chi-square statistic computed to test the joint insignificance of covariates reduced significantly after matching among truckers reporting sex with paid female partners (before matching: $\chi^{2}$ statistic $=99.6, p<0.001$; after matching: $\chi^{2}$ statistic $=4.6, p=0.948)$ as well as among those reporting sex with non-paid female partners (before matching: $\chi^{2}$ statistic $=74.4, p<0.001$; after matching: $\chi^{2}$ statistic $=11.3$, $p=0.418$ ). Similar results were obtained while comparing socio-demographic characteristics of all unexposed and matched unexposed truckers with the socio-demographic
Table 1 Socio-demographic characteristics, intervention exposure, and sexual behavior among truckers, India, $2009(N=2,723)$

\begin{tabular}{ll}
\hline $\begin{array}{l}\text { Socio-demographic characteristics, intervention } \\
\text { exposure, and sexual behavior }\end{array}$ & $\begin{array}{l}\text { Percentage and } \\
\text { mean }\end{array}$ \\
\hline Socio-demographic characteristics & \\
Mean age in years (SD) & $30.2(8.0)$ \\
Formal schooling & \\
Marital status & 83.4 \\
Currently married & \\
Not currently married & \\
Occupation & 33.3 \\
Driver & \\
Helper & 77.9 \\
Mean duration of working as & 22.1 \\
trucker in years (SD) & $8.4(6.5)$ \\
Route categories & \\
North-East & 20.1 \\
North-South & 20.6 \\
North-West & 31.2 \\
West-South & 11.8 \\
West-East & 16.2 \\
Living at native place & \\
Intervention Exposure & \\
No exposure & \\
Exposed only to mid-media & \\
Exposed only to mid-media and IPC & \\
Exposed only to mid-media and clinics & \\
Exposed to all three intervention components & \\
Exposed to any intervention & \\
Sexual behavior & \\
Sex with paid female partners in the past & \\
12 months & \\
Consistent condom use with paid female \\
partners
\end{tabular}

$S D$ standard deviation, IPC interpersonal communication

${ }^{a}$ Formal schooling refers to the ability to both read and write

b Not currently married includes truckers who were never married, divorced or widower

${ }^{c}$ Exposure to any intervention was defined as exposure to at least one of the three intervention components, namely, mid-media, IPC and project-run clinics in the past 12 months

d Among those who reported sex with paid female partners in the past 12 months

e Among those who reported sex with non-paid female partners in the past 12 months

characteristics of truckers exposed to different categories of intervention exposure (results not shown in tabular form but available upon request). 
Table 2 Truckers' sexual behavior by exposure to intervention components, India, $2009(N=2,723)$

\begin{tabular}{|c|c|c|c|c|c|c|c|c|}
\hline \multirow[b]{2}{*}{$\begin{array}{l}\text { Sexual behavior } \\
\text { in past } 12 \text { months }\end{array}$} & \multicolumn{3}{|c|}{ Exposure to any intervention $^{\mathrm{a}}$} & \multicolumn{5}{|c|}{ Categories of intervention exposure } \\
\hline & $\begin{array}{l}\text { No } \\
(N=991)\end{array}$ & $\begin{array}{l}\text { Yes } \\
(N=1,732)\end{array}$ & $\begin{array}{l}p \text { value }^{\mathrm{b}} \text { (test } \\
\text { Statistic) }\end{array}$ & $\begin{array}{l}\text { Only mid- } \\
\text { media } \\
(\mathrm{N}=651)\end{array}$ & $\begin{array}{l}\text { Only mid- } \\
\text { media and } \\
\text { IPC } \\
(\mathrm{N}=188)\end{array}$ & $\begin{array}{l}\text { Only mid- } \\
\text { media and } \\
\text { project-run } \\
\text { clinic } \\
(\mathrm{N}=449)\end{array}$ & $\begin{array}{l}\text { All three } \\
\text { intervention } \\
\text { components } \\
(\mathrm{N}=444)\end{array}$ & $\begin{array}{l}p \text { value }^{\mathrm{c}} \text { (test } \\
\text { Statistic) }\end{array}$ \\
\hline $\begin{array}{l}\text { Sex with paid } \\
\text { female partners } \\
(\%)\end{array}$ & 28.3 & 53.3 & $<0.001(166.92)$ & 43.3 & 47.6 & 61.4 & 64.5 & $<0.001(229.91)$ \\
\hline $\begin{array}{l}\text { Sex with non- } \\
\text { paid female } \\
\text { partners }(\%)\end{array}$ & 16.1 & 25.0 & $<0.001(29.23)$ & 16.4 & 27.5 & 24.9 & 36.6 & $<0.001(93.29)$ \\
\hline $\begin{array}{l}\text { Consistent } \\
\text { condom use } \\
\text { with paid } \\
\text { female } \\
\text { partners }{ }^{\mathrm{d}}(\%)\end{array}$ & 65.8 & 76.5 & $<0.001(12.85)$ & 77.3 & 63.3 & 80.7 & 76.0 & $<0.001(23.56)$ \\
\hline $\begin{array}{l}\text { Consistent } \\
\text { condom use } \\
\text { with non-paid } \\
\text { female } \\
\text { partners }^{\mathrm{e}}(\%)\end{array}$ & 32.5 & 45.3 & $0.005(7.82)$ & 31.8 & 34.6 & 54.9 & 50.9 & $<0.001(24.67)$ \\
\hline
\end{tabular}

$I P C$ interpersonal communication

${ }^{a}$ Exposure to any intervention was defined as exposure to at least one of the three intervention components, namely, mid-media, IPC and project-run clinics in the past 12 months

${ }^{\mathrm{b}} p$ values are obtained by comparing percentages for those who were exposed to any intervention and those who were not exposed to any intervention using Person's $\chi^{2}$ test statistic. Estimated values of the $\chi^{2}$ statistic are given in brackets below the corresponding $p$-values

${ }^{c} p$ values are obtained by comparing percentages across categories of intervention exposure using $\chi^{2}$ statistic. Estimated values of the $\chi^{2}$ statistic are given in brackets below the corresponding $p$ values

d Among those who reported having sex with paid female partners in the past 12 months

e Among those who reported having sex with non-paid female partners in the past 12 months

\section{Impact of Intervention Exposure on Consistent Condom Use}

Table 4 shows the impact of truckers' exposure to different intervention components on consistent condom use with paid and non-paid female partners. When compared with no exposure, the exposure to any intervention increased consistent condom use with paid female partners by $7.2 \%$ (95\% confidence interval (CI): 4.5-16.9, $p=0.001)$ and consistent condom use with non-paid female partners by $12.4 \%$ (95\% CI: 4.3-21.2, $p=$ 0.003). Exposure to mid-media alone increased consistent condom use with paid female partners by $9.9 \%(95 \%$ CI; 4.0-18.9, $p=0.003$ ) as compared with no exposure; however, this was not found to have an impact on consistent condom use with non-paid female partners. Similarly, exposure to only mid-media and project-run clinics increased consistent condom use with paid female partners by $11.4 \%(95 \%$ CI: 8.2-21.5, $p<0.001)$ and consistent condom use with non-paid female partners by $26.0 \%$ (95 \% CI: 11.6-33.3, $p<0.001)$ when compared with no exposure.
The additional impact of project-run clinics on consistent condom use with paid female partners was not evident when compared with exposure to only mid-media. However, visits to project-run clinics increased consistent condom use with non-paid female partners by about $20 \%$ (95\% CI: $11.1-35.2, p<0.001$ ) as compared to exposure to mid-media alone. Similar results about the additional combined impact of IPC and project-run clinics were found while comparing exposure to all three components with exposure to mid-media alone.

\section{Sensitivity Analyses}

The estimated impact of truckers' exposure to any intervention on consistent condom use with paid female partners was insensitive to a bias introduced by unobserved confounders that increase the odds of exposure to any intervention up to $30 \%$ (Mantel-Haenszel statistic $=1.85$, $p=0.030$ ) (Table 5). Similarly, its impact on consistent condom use with non-paid female partners remained insensitive to the presence of an unobserved confounder that increased the odds of exposure up to $20 \%$ (Mantel-Haenszel 


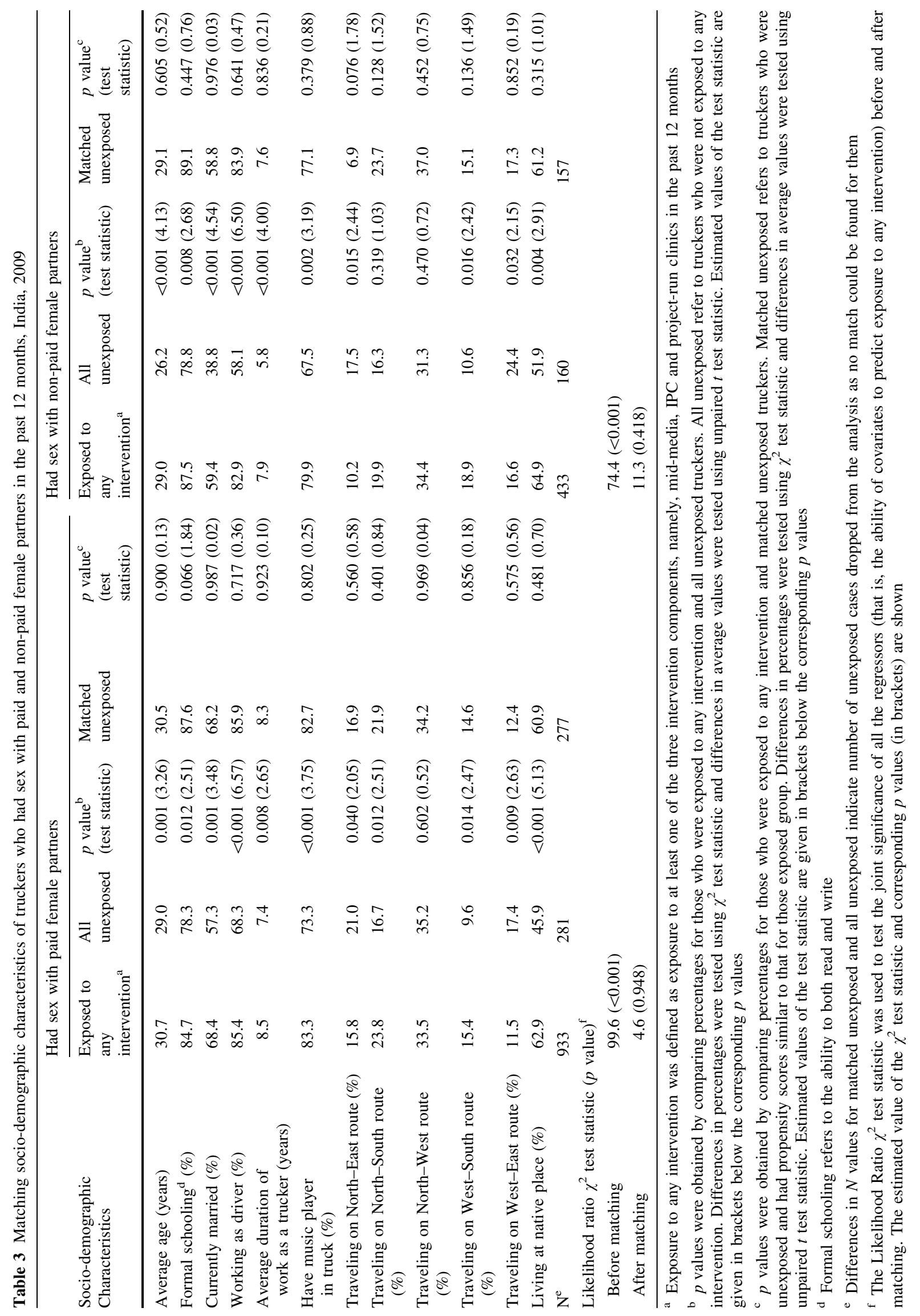




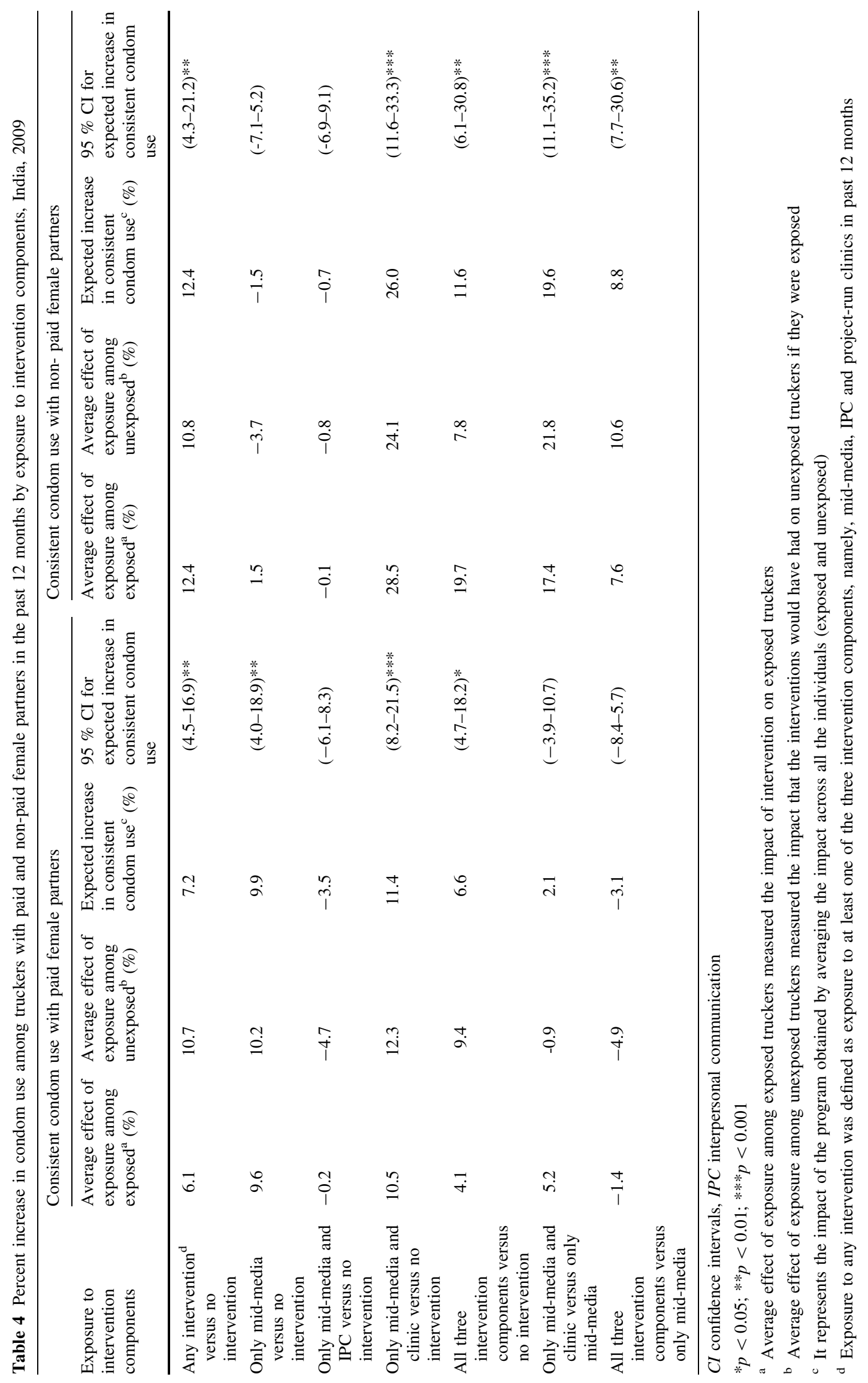


Table 5 Sensitivity analysis: Rosenbaum bounds for the estimated impact of exposure to intervention on consistent condom use with paid and non-paid female partners, India, 2009

\begin{tabular}{|c|c|c|c|c|}
\hline \multirow[t]{2}{*}{ Exposure to intervention components } & \multicolumn{2}{|c|}{$\begin{array}{l}\text { Consistent condom use with paid female } \\
\text { partners }\end{array}$} & \multicolumn{2}{|c|}{ Consistent condom use with non-paid female partners } \\
\hline & $\begin{array}{l}\text { Permissible odds of differential } \\
\text { exposure to intervention due to } \\
\text { unobserved factors }\end{array}$ & $\begin{array}{l}p \text { value }^{\text {a }} \text { (test } \\
\text { statistic) }\end{array}$ & $\begin{array}{l}\text { Permissible odds of } \\
\text { differential exposure to intervention } \\
\text { due to unobserved factors }\end{array}$ & $\begin{array}{l}p \text { value }^{\mathrm{a}} \\
\text { (test } \\
\quad \text { statistic) }\end{array}$ \\
\hline $\begin{array}{l}\text { Any intervention }{ }^{\mathrm{b}} \text { versus no } \\
\text { intervention }\end{array}$ & $30 \%$ & $0.032(1.85)$ & $20 \%$ & $0.044(1.71)$ \\
\hline $\begin{array}{l}\text { Only mid-media versus no } \\
\text { intervention }\end{array}$ & $30 \%$ & $0.048(1.66)$ & NA & NA \\
\hline $\begin{array}{l}\text { Only mid-media and project-run } \\
\text { clinic versus no intervention }\end{array}$ & $50 \%$ & $0.029(1.89)$ & $60 \%$ & $0.038(1.77)$ \\
\hline $\begin{array}{l}\text { All three intervention components } \\
\text { versus no intervention }\end{array}$ & $20 \%$ & $0.034(1.82)$ & $30 \%$ & $0.041(1.74)$ \\
\hline $\begin{array}{l}\text { Only mid-media and clinic versus } \\
\text { only mid-media }\end{array}$ & NA & NA & $50 \%$ & $0.033(1.82)$ \\
\hline $\begin{array}{l}\text { All three intervention components } \\
\text { versus only mid-media }\end{array}$ & NA & NA & $30 \%$ & $0.044(1.73)$ \\
\hline
\end{tabular}

NA: The sensitivity analyses were not done as exposure to the corresponding components of intervention did not result in a significant difference in condom use with paid female partners and non-paid female partners

${ }^{a} p$ values were estimated using Mantel-Haenszel statistic under the assumption of overestimation of the impact of exposure to corresponding component of intervention. Estimated values of Mantel-Haenszel test statistic are given in brackets below the corresponding $p$ values

${ }^{\mathrm{b}}$ Exposure to any intervention was defined as exposure to at least one of the three intervention components, namely, mid-media, IPC and project-run clinics in the past 12 months

statistic $=1.71, p=0.044)$. Similar results were obtained for estimated impact of exposure to different categories of intervention exposure on consistent condom use with paid and non-paid female partners.

\section{Discussion}

The study findings showed differential effects of exposure to components of the Kavach intervention on consistent condom use with paid and non-paid female partners. For instance, trucker's exposure to mid-media alone as compared to those who were not exposed to any intervention reported significantly higher consistent condom use with paid female partners. Whereas, truckers who visited project-run clinics as compared to those with exposure to midmedia alone have reported higher consistent condom use with non-paid female partners. Exposure only to midmedia and IPC, without being supplemented by visits to project-run clinics, did not have a significant impact on condom use behavior.

The finding that truckers' exposure to mid-media alone has increased consistent condom use with paid female partners can be explained, to some extent, by the possible confounding effect of parallel intensive interventions among FSWs in several states of India [15]. The national AIDS control program as well as the Avahan supported initiatives for the social marketing of condoms at 'hotspots' in high HIV prevalence states in India have increased the availability of condoms in high risk settings where commercial sex takes place [28, 40]. In the context of several intervention programs to promote condom use in commercial sex, information on the availability and importance of condom use imparted through the midmedia could lead to high rates of consistent condom use with paid female partners. However, as the study findings indicate, exposure to mid-media alone did not increase consistent condom use with non-paid female partners, which may perhaps be explained by two factors. First, the degree of intimacy and trust is higher with non-paid female partners than paid partners, and hence condom use with such partners is low [14-16]. Second, noncommercial sex takes place in settings (e.g., homes) where the reach and effectiveness of HIV prevention programs may be limited.

Information on safe sexual practices provided by midmedia events coupled with counseling by a qualified counselor on safe sexual practices, correct and consistent condom use and treatment of STI by a qualified doctor in project-run clinics appear to be the most effective package of services to increase condom use with both paid and nonpaid female partners. Findings suggest that visits to projectrun clinics did not have an additional impact as compared to exposure to mid-media alone in terms of consistent 
condom use with paid female partners. However, visits to project-run clinics had an additional impact on consistent condom use with non-paid female partners compared to exposure to mid-media alone. One possible reason behind success of project-run clinics in increasing consistent condom use with non-paid female partners could be the in-depth one-to-one discussions with qualified counselors and qualified doctors. Research from developing countries indicate that HIV counseling recipients are more likely to use condom than those who do not receive it [41]. A recent study among cross-border truck drivers in Hong Kong also found voluntary HIV counseling and testing plus the information dissemination approach to be more effective in promoting safe sex behaviors than the information dissemination approach alone [42]. Although further studies are needed to understand the processes that motivate truckers to use condoms with non-paid female partners, this paper suggests that the provision of clinics with integrated one-to-one counseling by trained professionals promotes consistent condom use, particularly with non-paid female partners.

Findings also indicate that as compared to no exposure, exposure to only mid-media and IPC did not improve condom use behavior. This could be due to the small sample size of truckers exposed to these two intervention components, as well as the fact that truckers who were exposed to only mid-media and IPC constitute a subgroup who did not visit the project-run clinics even after being exposed to two intervention components that were aimed to motivate them to visit these clinics. As this group of truckers could not be convinced to visit clinics even after repeated efforts, it may be argued that these truckers did not give much importance to the messages on condom use provided during mid-media events and IPC sessions. Hence, though covered by the program, these truckers' condom use behavior was similar to those who were not exposed to any intervention component. These findings suggest the need to change the IPC approach (e.g., using photographs to illustrate the consequences of STI) to motivate truckers to use condoms with non-regular female sexual partners.

The percentage of truckers exposed to the intervention as observed in this study was found to be higher than that found in a large-scale survey among long distance truck drivers conducted during 2009-10 in India [15]. This may be because our study was limited to transshipment locations where the Kavach intervention program was being implemented whereas the above-mentioned study included transshipment locations where this program did not operate.

On the other hand, the levels of consistent condom use with paid female partners $(74.1 \%)$ and non-paid female partners $(41.8 \%)$ as found in this study are consistent with other large-scale study among long distance truckers in India which found that about $74 \%$ of the truck drivers used condoms consistently with paid female partners whereas about $37 \%$ of them used condom consistently with nonpaid female partners [15]. In recent past, small-scale studies in different parts of the country have found a wide range (57\%-84\%) of estimates for consistent condom use by truckers with paid female partners. This wide variation in estimates across the small-scale studies are often due to small sample size, and variation in geographic region. Differentials in sexual behaviors of truckers from different parts of the country have been documented elsewhere $[15,35]$.

This study has some limitations, which should be considered when interpreting the results. First, findings are based on self- reports, which are vulnerable to social desirability and recall biases. The survey measured a limited number of socio-demographic characteristics and did not include some of the important variables such as income and alcohol consumption, which may confound the results. Finally, although the PSM approach was used to establish a causal relationship between exposure to intervention and consistent condom use, this should not be considered an alternative approach to randomized trials with valid case and control groups.

In conclusion, the study documents that the Kavach intervention resulted in a significant increase in consistent condom use with both paid and non-paid female partners. Exposure to mid-media alone substantially increased consistent condom use by truckers with paid female partners whereas exposure to project-run clinics had a significant impact on increasing condom use with non-paid female partners. These findings suggest the need for intensive midmedia events to increase awareness of HIV and easily accessible clinics that provide counseling on safe sex practices and STI treatment services to truckers to increase the adoption of consistent condom use with non-regular female partners. It also highlights the need to understand truckers' risk perceptions, needs and the barriers to the uptake of project-run clinic services even after repeated attempts to promote behavior change.

Acknowledgments This paper was written as part of a mentorship programme under the Knowledge Network project of the Population Council, which is a grantee of the Bill \& Melinda Gates Foundation through Avahan, its India AIDS Initiative. The views expressed herein are those of the authors and do not necessarily reflect the official policy or position of the Bill \& Melinda Gates Foundation and Avahan.

Open Access This article is distributed under the terms of the Creative Commons Attribution License which permits any use, distribution, and reproduction in any medium, provided the original author(s) and the source are credited. 


\section{References}

1. Alam N, Rahman M, Gausia K, Yunus MD, Islam N, Chaudhury $\mathrm{P}$, et al. Sexually transmitted infections and risk factors among truck stand workers in Dhaka, Bangladesh. Sex Transm Dis. 2007;34(2):99-103.

2. Chen XS, Yin YP, Gong XD, Liang GJ, Zhang WY, Poumerol G, et al. Prevalence of sexually transmitted infections among longdistance truck drivers in Tongling, China. Int J STD AIDS. 2006;17(5):304-8.

3. Podhisita C, Wawer MJ, Pramualratana A, Kanungsukkasem U, McNamara R. Multiple sexual partners and condom use among long-distance truck drivers in Thailand. AIDS Educ Prev. 1996;8(6):490-8.

4. Wong WC, Tam SM, Leung PW. Cross-border truck drivers in Hong Kong: their psychological health, sexual dysfunctions and sexual risk behaviors. J Travel Med. 2007;14(1):20-30.

5. Atilola GO, Akpa OM, Komolafe IO. HIV/AIDS and the longdistance truck drivers in south-west Nigeria: a cross-sectional survey on the knowledge, attitude, risk behaviour and beliefs of truckers. J Infect Public Health. 2010;3(4):166-78.

6. Ekanem EE, Afolabi BM, Nuga AO, Adebajo SB. Sexual behaviour, HIV-related knowledge and condom use by intra-city commercial bus drivers and motor park attendants in Lagos, Nigeria. Afr J Reprod Health. 2005;9(1):78-87.

7. Sunmola AM. Sexual practices, barriers to condom use and its consistent use among long distance truck drivers in Nigeria. AIDS Care. 2005;17(2):208-21.

8. Ferguson AG, Morris CN. Mapping transactional sex on the Northern Corridor highway in Kenya. Health Place. 2007;13(2): 504-19.

9. Lippman SA, Pulerwitz J, Chinaglia M, Hubbard A, Reingold A, Diaz J. Mobility and its liminal context: exploring sexual partnering among truck drivers crossing the Southern Brazilian border. Soc Sci Med. 2007;65(12):2464-73.

10. Sorensen W, Anderson PB, Speaker R, Vilches JE. Assessment of condom use among Bolivian truck drivers through the lens of social cognitive theory. Health Promot Int. 2007;22(1):37-43.

11. Lichtenstein B, Hook EW 3rd, Grimley DM, St Lawrence JS, Bachmann LH. HIV risk among long-haul truckers in the USA. Cult Health Sex. 2008;10(1):43-56.

12. McCree DH, Cosgrove S, Stratford D, Valway S, Keller N, VegaHernandez J, et al. Sexual and drug use risk behaviors of longhaul truck drivers and their commercial sex contacts in New Mexico. Public Health Rep. 2010;125(1):52-60.

13. Orubuloye IO, Caldwell P, Caldwell JC. The role of high-risk occupations in the spread of AIDS: truck drivers and itinerant market women in Nigeria. Int Fam Plan Perspect. 1993;19(2): 43-48, 71.

14. Dude A, Oruganti G, Kumar V, Mayer KH, Yeldandi V, Schneider JA. HIV Infection, genital symptoms and sexual risk behavior among indian truck drivers from a large transportation company in South India. J Glob Infect Dis. 2009;1(1):21-8.

15. Pandey A, Mishra RM, Sahu D, Benara SK, Sengupta U, Paranjape RS, et al. Heading towards the safer highways: an assessment of the Avahan prevention programme among long distance truck drivers in India. BMC Public Health. 2011;11 (suppl 6):S15.

16. Sanjeev K, Garg SK, Bajpai SK. A study of knowledge, sexual behaviour and practices regarding HIV/AIDS among long distance truck drivers. Indian J Public Health. 2009;53(4):243-5.

17. Manjunath JV, Thappa DM, Jaisankar TJ. Sexually transmitted diseases and sexual lifestyles of long-distance truck drivers: a clinico-epidemiologic study in South India. Int J STD AIDS. 2002;13(9):612-7.
18. Bal B, Ahmed SI, Mukherjee R, Chakraborty S, Niyogi SK, Talukder A, et al. HIV infection among transport workers operating through Siliguri-Guwahati national highway, India. J Int Assoc Physicians AIDS Care (Chic). 2007;6(1):56-60.

19. The Communication Initiative Network. HIV/AIDS Workplace and Local Community Education Programme-India. http://www.comminit.com/en/node/118715. Accessed 10 Jan 2012.

20. Raman S. Positive reinforcement to promote safer sex among clients. AIDS Health Promot Exch. 1992;1:6-9.

21. Singh YN, Singh K, Joshi R, Rustagi GK, Malaviya AN. HIV infection among long-distance truck drivers in Delhi, India. J Acquir Immune Defic Syndr. 1993;6(3):323.

22. Bhoruka Public Welfare Trust. U Turn. Kolkata. http://www. bpwt.org/publications.asp. Accessed 25 Dec 2010.

23. Majumdar A. Halting AIDS on highways. In: Raju S, Leonard A, editors. Men as supportive partners in reproductive health: moving from rhetoric to reality. New Delhi: Population Council; 2000.

24. World Health Organization. Integrating gender into HIV/AIDS programs: a review paper. Geneva: Department of Gender and Women's Health, Family and Community Health. 2003.

25. National AIDS Control Organization (NACO). National AIDS control programme, Phase II. New Delhi: Ministry of Health \& Family Welfare, Government of India. 1999.

26. Bill \& Melinda Gates Foundation (BMGF). Off the beaten track: Avahan's experience in the business of HIV prevention among india's long-distance truckers. New Delhi: Bill \& Melinda Gates Foundation. 2008.

27. Chandrasekaran P, Dallabetta G, Loo V, Rao S, Gayle H, Alexander A. Containing HIV/AIDS in India: the unfinished agenda. Lancet Infect Dis. 2006;6(8):508-21.

28. Bill \& Melinda Gates Foundation (BMGF). Avahan, the India AIDS initiative - the business of HIV prevention at scale. New Delhi: Bill \& Melinda Gates Foundation. 2008.

29. Ritzer George. The Mcdonaldization of society. J Am Cult. 1983;6(1):100-7.

30. Williamson E, Morley R, Lucas A, Carpenter J. Propensity scores: from naive enthusiasm to intuitive understanding. Stat Methods Med Res. 2012;21(3):273-93.

31. Rubin DB, Thomas N. Matching using estimated propensity scores: relating theory to practice. Biometrika. 1996;52(1): 249-64.

32. Rosenbaum PR, Rubin DB. The central role of the propensity score in observational studies for causal effects. Biometrika. 1983;70(1):41-55.

33. Dehejia R. Practical propensity score matching: a reply to Smith and Todd. J Econ. 2005;125:355-364.

34. Mahal A, Canning D, Odumosu K, Okonkwo P. Assessing the economic impact of HIV/AIDS on Nigerian households: a propensity score matching approach. AIDS. 2008;22(Suppl 1): S95-101.

35. Pandey A, Benara SK, Roy N, Sahu D, Thomas M, Joshi DK, et al. Risk behaviour, sexually transmitted infections and HIV among long-distance truck drivers: a cross-sectional survey along national highways in India. AIDS. 2008;22(Suppl 5):S81-90.

36. Chaturvedi S, Singh Z, Banerjee A, Khera A, Joshi RK, Dhrubajyoti D. Sexual behaviour among long distance truck drivers. Indian J Community Med. 2006;31(3):153-6.

37. Stuart EA. Matching methods for causal inference: a review and a look forward. Stat Sci. 2010;25(1):1-21.

38. Austin PC, Grootendorst P, Anderson GM. A comparison of the ability of different propensity score models to balance measured variables between treated and untreated subjects: a Monte Carlo study. Stat Med. 2007;26:734-53. 
39. Rosenbaum PR, Rubin DB. Constructing a control group using multivariate matched sampling methods that incorporate the propensity score. Am Stat. 1985;39:33-8.

40. National AIDS Control Organization (NACO). NACP-III-to halt and reverse the HIV epidemic in India. New Delhi Ministry of Health \& Family Welfare, Government of India. 2006.

41. Denison JA, O'Reilly KR, Schmid GP, Kennedy CE, Sweat MD. HIV voluntary counseling and testing and behavioral risk reduction in developing countries: a meta-analysis, 1990-2005. AIDS Behav. 2008;12(3):363-73.

42. Lau JT, Tsui HY, Cheng S, Pang M. A randomized controlled trial to evaluate the relative efficacy of adding voluntary counseling and testing (VCT) to information dissemination in reducing HIV-related risk behaviors among Hong Kong male cross-border truck drivers. AIDS Care. 2010;22(1):17-28. 